\title{
The prevalence of fatigue and associated health and safety risk factors among taxi drivers in Singapore
}

See Ming $\underline{\operatorname{Lim}}^{1}$, MBBS, MPH, Sin Eng $\underline{\text { Chia }}^{2}$, MBBS, MD

INTRODUCTION Driver fatigue is one of the biggest health and safety concerns within the road transport sector. This study aimed to determine the prevalence of fatigue among taxi drivers in Singapore, to better understand the general working and health conditions of this group of people, and to determine the risk factors associated with fatigued driving. METHODS A total of 340 taxi drivers were randomly selected for participation in a self-administered questionnaire survey, which included height and weight measurements. The response rate was $68.2 \%$. The survey consisted of four main categories: personal particulars; social habits; work patterns; and sleep profile. The Epworth Sleepiness Scale was used to estimate the level of daytime sleepiness and fatigue. Multiple logistic regression analysis was used to estimate the adjusted odd ratios and 95\% confidence intervals associated with risk factors related to fatigue among taxi drivers. RESULTS A high proportion of taxi drivers were obese and had self-reported hypertension, diabetes mellitus and high cholesterol. Driver fatigue was associated with very poor/poor self-rating of quality of sleep, having an additional parttime job, drinking three or more caffeinated drinks daily, and driving more than 10 hours a day.

CONCLUSION We hope that the findings of the present study will improve awareness of the work and health conditions of taxi drivers, and contribute toward efforts to achieve a healthier workforce. A lower prevalence of fatigued driving may lead to lower risks of road traffic accidents, decreased economic loss, increased productivity, and safer roads for all.

Keywords: fatigue, health, safety, taxi drivers

\section{INTRODUCTION}

Fatigue is a state of tiredness that results in reduced mental and/ or physical performance. It can decrease a person's alertness and compromise a person's motor skills, reflex ability, judgement and decision-making. In simple terms, fatigue has been generally described as being sleepy, tired or exhausted..$^{(1,2)}$

One of the biggest growing health and safety concerns within the road transport sector today is fatigued driving. Driving when fatigued can lead to impaired performance at the wheel and can ultimately result in the driver falling asleep at the wheel. Fatigued driving is a workplace hazard that causes thousands of road traffic accidents and fatalities each year. Studies conducted all over the world have shown that fatigued driving contributes to approximately $3 \%-30 \%$ of vehicle crashes. ${ }^{(3)}$ In the United States, more than 1,500 people die yearly due to road traffic accidents caused by fatigued driving; another 71,000 are injured as a result of these accidents and the economic loss due to such accidents is estimated to be USD12.5 billion. ${ }^{(4)}$ The prevalence of road traffic accidents as a result of fatigued driving is likely to be under-reported because there is often a lack of concrete evidence to suggest the cause was fatigued driving (e.g. the driver might not report it due to potential liabilities). ${ }^{(5)}$

Fatigue can cause the following symptoms and effects among drivers: loss of alertness, difficulty keeping eyes in focus, frequent yawning, reduced awareness of surroundings, loss of concentration, drifting out of the lane, missing a turnoff, microsleep, and near-accidents/road traffic accidents. ${ }^{(6)}$ There are many possible causes of fatigue. Increasing age is a well-known contributory cause of fatigue. Ageing leads to fatigue in several ways: (a) deterioration of physiological and biological systems, resulting in lowered performance due to altered eyesight, longer reaction time and decreased muscle strength; (b) difficulty in adjusting to circadian rhythms due to shift work; and (c) inability to adjust to long working hours of 12 hours or more. The critical age is suggested to be around 50 years, which is approximately the time when complaints associated with sleep quality and fatigue are most prominent. ${ }^{(7)}$

It has also been documented that some medical conditions contribute to fatigue among drivers. One such medical condition is obesity. Compared to non-obese drivers, obese drivers have been found to be more prone to fatigue while driving and more likely to encounter a road traffic accident. ${ }^{(8)}$ It has been postulated that long hours of driving, limited food options and irregular schedules have led to a high risk of obesity among commercial drivers. ${ }^{(8)}$ Obesity is commonly associated with obstructive sleep apnoea (OSA), a sleep disorder that is characterised by disrupted and non-restorative sleep, which may lead to excessive daytime fatigue and sleepiness. Persons with OSA often display symptoms such as snoring, breathing pauses and choking during sleep. Both the number of episodes of sleepiness when driving and the number of road traffic accidents have been found to be significantly greater in drivers with OSA than in controls. ${ }^{(9)}$ Other medical conditions such as anaemia, chronic infection, depression, drug dependence and ongoing pain are also welldocumented causes of fatigue. ${ }^{(6)}$

Excessive caffeine intake can cause insomnia, which can lead to fatigue. Caffeine reduces both slow-wave sleep (the most restorative part of sleep) and rapid eye movement sleep in the

${ }^{1}$ Preventive Medicine Residency Programme, National University Health System, ${ }^{2}$ Saw Swee Hock School of Public Health, National University of Singapore, Singapore Correspondence: Dr Lim See Ming, Senior Resident, National University Health System, National University Hospital, 1E Kent Ridge Road, Tower Block, Level 6, Singapore 119228. seeming.lim@mohh.com.sg 
sleep cycle. It also increases the period of wakefulness and can lengthen the time taken to fall asleep at night if large amounts of caffeine are taken in the evening. ${ }^{(10)}$ In general, more than $200 \mathrm{mg}$ of caffeine (approximately three or more caffeinated drinks) needs to be consumed in order for sleep to be significantly affected.

It is well known that both lack of sleep and poor quality of sleep contribute to fatigue. The average sleep requirement for an adult is around 7-8 hours a day. Without adequate rest, drivers are likely to be fatigued the next day. If the sleep debt accumulates and becomes too large, microsleep will happen. ${ }^{(6)}$ Driver alertness is known to decrease during the time periods 0100-0800 hours and 1400-1800 hours, with the decrease being less significant in the latter time period. ${ }^{(11)}$ This is due to the effect of the intrinsic circadian clock of the human body on performance and activity during shift work. ${ }^{(11)}$

Long hours of driving per day with minimal rest breaks in between are risk factors to fatigued driving, ${ }^{(11)}$ although the duration of rest breaks does not seem to reduce driver fatigue. ${ }^{(11)}$ The International Labour Organization's hours of work and rest periods convention (for road transport) states that the maximum driving total time should not exceed 9 hours daily or 48 hours weekly, with every driver entitled to a break after 4 hours of continuous driving. ${ }^{(12)}$ A maximum 10-hour driving limit is imposed in the United States and the United Kingdom.

In Singapore, there are currently no official guidelines on the work hours and rest periods of taxi drivers. Since fatigued driving continues to be a growing issue within Singapore's transport sector, we conducted a study on the prevalence of fatigue among taxi drivers, to better understand the general working and health conditions of this group of people, as well as to determine the risk factors associated with fatigued driving.

\section{METHODS}

The present study was conducted at one of the largest local taxi companies. In 2012, the company had a fleet of nearly 3,500 taxis, forming around $30 \%$ of all taxis in Singapore at that time. This study was approved by the Institutional Review Board of the National University of Singapore, and permission to conduct the survey was granted by the taxi company.

A total of 340 taxi drivers were randomly selected to participate in this self-administered questionnaire survey at the company during office hours. The taxi drivers were there for maintenance or repair of their taxis, and/or to pay their monthly rental fees. While the taxi drivers were in the queue, we approached alternate drivers to participate in the survey. Informed consent was obtained before the survey was administered. Of the 340 taxi drivers approached, 232 agreed to participate in the survey, giving a response rate of $68.2 \%$. Of these 232 taxi drivers, 225 completed both the survey and measurement of height and weight, while 6 completed only the survey. One taxi driver did not complete the survey due to time constraints, and was therefore excluded in the analysis of the results.

The questionnaire survey forms were administered in both English and Mandarin. Each survey form consisted of four main parts: (a) personal particulars; (b) social habits; (c) work pattern and sleep profile; and (d) a validated questionnaire (i.e. Epworth Sleepiness scale [ESS] $)^{(13,14)}$ to estimate usual daytime sleepiness. The ESS was used as an indicator of the level of fatigue. The Mandarin version of the ESS used in the present study had been validated by previous studies. ${ }^{(13,14)}$ The height and weight of the taxi drivers were measured after the completion of the survey forms. These measurements were taken for the computation of body mass index (BMI). Questions on social habits, work pattern and sleep profile were used to examine the various identified risk factors of fatigue among taxi drivers. These risk factors included number of caffeinated drinks daily, shift status, average hours of driving per day, number of rest days per week, number of rest breaks per shift, time spent at each rest break, additional part-time job, hours of sleep prior to work, self-rating of quality of sleep, and presence of a triad of sleep apnoea symptoms (i.e. witnessed snoring, breathing pauses and choking).

For statistical analysis, the age groups were categorised into 31-50 years and $51-70$ years, as age above 50 years was shown to be associated with fatigue and poor sleep quality. ${ }^{(7)} \mathrm{BMI}$ was classified into two groups to reflect the non-obese and obese participants according to Asian standards $\left(<27.5 \mathrm{~kg} / \mathrm{m}^{2}\right.$ and $\geq 27.5 \mathrm{~kg} / \mathrm{m}^{2}$, respectively). ${ }^{(15)}$ The number of caffeinated drinks taken per day was categorised into $0-2$ and $\geq 3$ drinks, as the approximate amount of caffeine needed to affect sleep has been shown to be $\geq 3$ caffeinated drinks per day (i.e. $\geq 3$ caffeinated drinks will likely exceed the $200 \mathrm{mg}$ limit of caffeine intake that will significantly affect sleep). ${ }^{(10)}$ Due to the effect of the intrinsic circadian clock on human performance and activity, ${ }^{(11)}$ the shift statuses were grouped to reflect the periods of decreased alertness (i.e. afternoon/overnight/24-hour shift or other shift timings). The following indicators of driver fatigue among the taxi drivers were used: > 10 hours of driving per day; $\leq 2$ rest breaks per shift; and $<7$ hours of sleep prior to work. ${ }^{(6,11,12)}$

To estimate usual daytime sleepiness, the participants were asked to rate the likelihood (never, slight, moderate or high) that they would fall asleep in each of the eight common situations listed in the ESS. Total Epworth score ranged from 0 to 24, with a score $<10$ considered normal, and a score $\geq 10$ indicating some level of daytime sleepiness and fatigue. ${ }^{(13)}$ STATA 12.1 data analysis and statistical software (StataCorp, College Station, TX, USA) was used for all statistical analyses. Adjusted odd ratios of the risk factors associated with driver fatigue were estimated using multiple logistic regression. A p-value $<0.05$ was considered statistically significant.

\section{RESULTS}

Table I shows the characteristics of the taxi drivers who participated in the study, while Table II shows the health conditions and associated risk factors of the taxi drivers as compared to the general adult population, according to National Health Survey (NHS) 2010. ${ }^{(16)}$ Table III shows the characteristics of the taxi drivers with an Epworth score $<10$ (controls, $\mathrm{n}=155$ ) and those with an Epworth score $\geq 10$ (cases, $n=76$ ), while Table IV shows the association between the various risk factors and fatigue (graded using the Epworth scale). 
Table I. Characteristics of the survey population $(n=231)$.

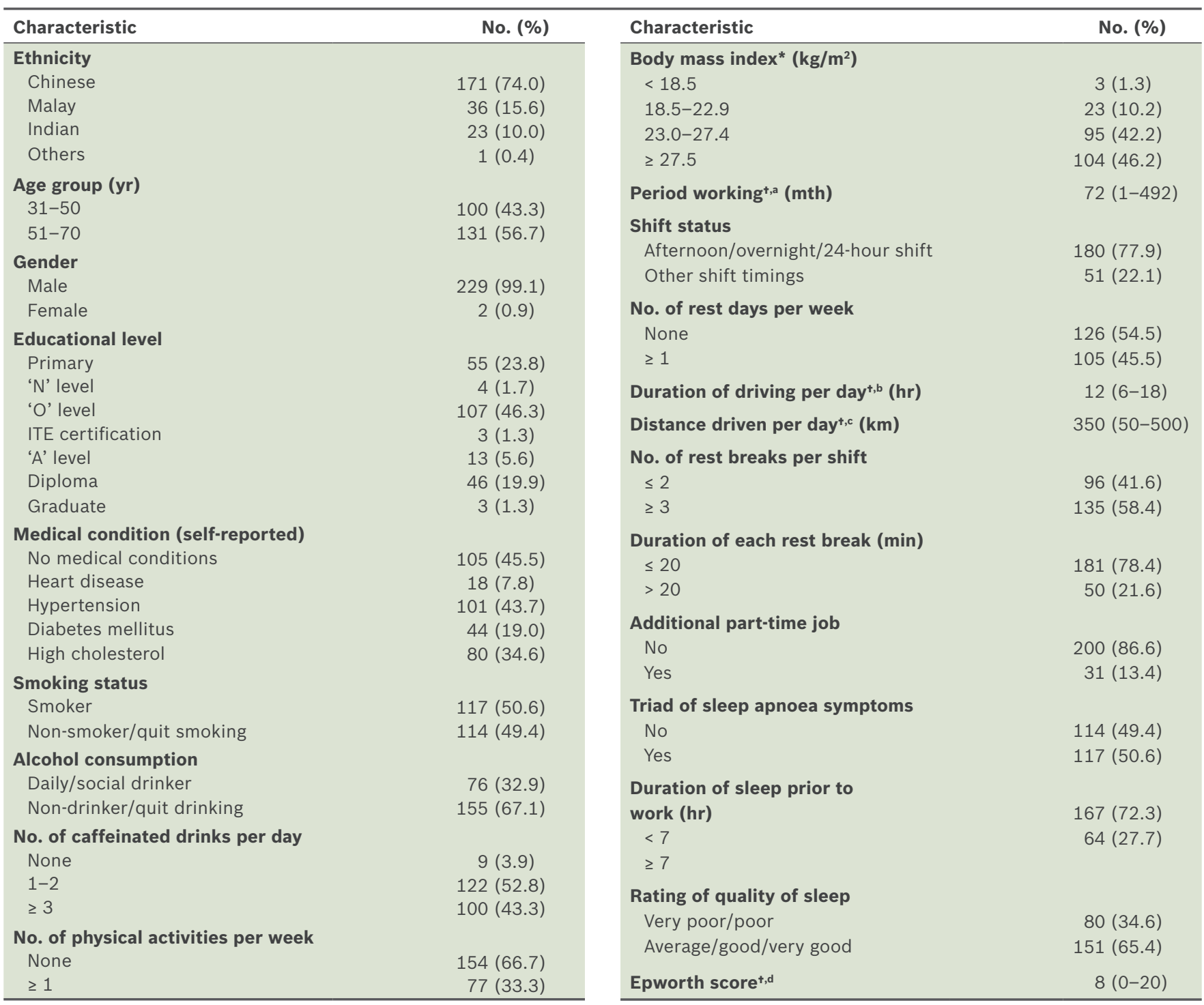

* Percentages calculated using a sample size of 225 taxi drivers, as 6 of the 231 taxi drivers declined height and weight measurements. + Data presented as median (range). aMean period working was 99.0 months. ${ }^{b}$ Mean duration of driving per day was 12.3 hours. ${ }^{c}$ Mean distance driven per day was 335.9 km. ${ }^{\mathrm{d}}$ Mean Epworth score was 8.0. ITE: Institute of Technical Education

Table II. Health conditions and associated risk factors of taxi drivers as compared to the adult population.

\begin{tabular}{lcc}
\hline Parameter & \multicolumn{2}{c}{ Percentage } \\
\cline { 2 - 3 } & $\begin{array}{c}\text { Proportion } \\
\text { of taxi } \\
\text { drivers }\end{array}$ & $\begin{array}{c}\text { Prevalence } \\
\text { in adult } \\
\text { population* }\end{array}$ \\
\hline Health condition (self-reported) & & \\
Hypertension & 43.7 & 23.5 \\
Diabetes mellitus & 19.0 & 11.3 \\
High cholesterol & 34.6 & 17.4 \\
Risk factor & & \\
Smoker & 50.6 & 16.3 \\
Physically inactive $_{\text {BMI }} \geq 27.5 \mathrm{~kg} / \mathrm{m}^{2}$ & 66.7 & 54.0 \\
\hline
\end{tabular}

* Data for adult population was obtained from National Health Survey 2010.(16) tBMI of only 225 taxi drivers was collected. BMI: body mass index

The proportion of ethnicity in our survey population $(74.0 \%$ Chinese, $15.6 \%$ Malay, $10.0 \%$ Indian and $0.4 \%$ others) closely resembled that of the general population of Singapore residents
(74.2\% Chinese, 13.3\% Malay, 9.2\% Indian and 3.3\% others, according to the Department of Statistics, Singapore, as at June 2012). ${ }^{(17)}$ The majority (99.1\%) of taxi drivers who participated in the study were male and most $(71.8 \%)$ had an educational level of ' $\mathrm{O}$ ' level and below (Table I). The proportion of taxi drivers who had self-reported medical conditions (i.e. hypertension, diabetes mellitus and high cholesterol) and associated risk factors (i.e. smoker, physically inactive and $\mathrm{BMI} \geq 27.5 \mathrm{~kg} / \mathrm{m}^{2}$ ) was higher than the prevalence reported in the general adult population in Singapore (Table II). ${ }^{(16)}$

The prevalence of sleepiness and fatigue among taxi drivers was around $32.9 \%$ (sleepiness and fatigue was defined as having an Epworth score $\geq 10$ ). We observed that fatigue and sleepiness was positively associated with a poor/very poor self-reported rating of quality of sleep, the presence of an additional part-time job, the consumption of $\geq 3$ caffeinated drinks per day and driving for $>10$ hours per day (Table III). Older age (i.e. 51-70 years), higher BMI (i.e. $\geq 27.5 \mathrm{~kg} / \mathrm{m}^{2}$ ), afternoon/overnight/24-hour shift 
Table III. Characteristics of taxi drivers with an Epworth score $<10$ ( $n=155)$ and taxi drivers with an Epworth score $\geq 10(n=76)$.

\begin{tabular}{|c|c|c|c|}
\hline \multirow[t]{2}{*}{ Characteristic } & \multicolumn{2}{|c|}{ No. (\%) } & \multirow[t]{2}{*}{ p-value } \\
\hline & $\begin{array}{c}\text { Epworth } \\
\text { score }<10 \\
(n=155)\end{array}$ & $\begin{array}{c}\text { Epworth } \\
\text { score } \geq 10 \\
(n=76)\end{array}$ & \\
\hline Ethnicity & & & NS \\
\hline Chinese & $120(77.4)$ & $51(67.1)$ & \\
\hline Malay & $21(13.5)$ & 15 (19.7) & \\
\hline Indian & $13(8.4)$ & $10(13.2)$ & \\
\hline Others & $1(0.6)$ & $0(0)$ & \\
\hline Age group (yr) & & & $<0.05$ \\
\hline $31-50$ & $51(32.9)$ & $49(64.5)$ & \\
\hline $51-70$ & $104(67.1)$ & $27(35.5)$ & \\
\hline Gender & & & NS \\
\hline Male & $154(99.4)$ & 75 (98.7) & \\
\hline Female & $1(0.6)$ & $1(1.3)$ & \\
\hline Education level & & & NS \\
\hline Primary & $47(30.3)$ & $8(10.5)$ & \\
\hline 'N' level & $2(1.3)$ & $2(2.6)$ & \\
\hline 'O' level & $73(47.1)$ & $34(44.7)$ & \\
\hline ITE certification & $1(0.6)$ & $2(2.6)$ & \\
\hline 'A' level & $12(7.7)$ & $1(1.3)$ & \\
\hline Diploma & $18(11.6)$ & $28(36.8)$ & \\
\hline Graduate & $2(1.3)$ & $1(1.3)$ & \\
\hline Body mass index* $\left(\mathrm{kg} / \mathrm{m}^{2}\right)$ & & & $<0.05$ \\
\hline$<27.5$ & $76(50.7)$ & $45(60.0)$ & \\
\hline$\geq 27.5$ & $74(49.3)$ & $30(40.0)$ & \\
\hline No. of caffeinated drinks per day & & & $<0.05$ \\
\hline $0-2$ & $98(63.2)$ & $33(43.4)$ & \\
\hline$\geq 3$ & $57(36.8)$ & $43(56.6)$ & \\
\hline Shift status & & & $<0.05$ \\
\hline Afternoon/overnight/24-hr shift & $111(71.6)$ & $69(90.8)$ & \\
\hline Other shift timings & $44(28.4)$ & $7(9.2)$ & \\
\hline No. of rest days per week & & & $<0.05$ \\
\hline None & $70(45.2)$ & $56(73.7)$ & \\
\hline$\geq 1$ & $85(54.8)$ & $20(26.3)$ & \\
\hline Duration of driving per day (hr) & & & $<0.05$ \\
\hline$\leq 10$ & $38(24.5)$ & $11(14.5)$ & \\
\hline$>10$ & $117(75.5)$ & $65(85.5)$ & \\
\hline No. of rest breaks per shift & & & NS \\
\hline$\leq 2$ & $64(41.3)$ & $32(42.1)$ & \\
\hline$\geq 3$ & $91(58.7)$ & $44(57.9)$ & \\
\hline $\begin{array}{l}\text { Duration of each rest } \\
\text { break (min) }\end{array}$ & & & $<0.05$ \\
\hline$\leq 20$ & $112(72.3)$ & $64(84.2)$ & \\
\hline$>20$ & $43(27.7)$ & $12(15.8)$ & \\
\hline Additional part-time job & & & $<0.05$ \\
\hline No & $148(95.5)$ & $52(68.4)$ & \\
\hline Yes & $7(4.5)$ & $24(31.6)$ & \\
\hline Triad of sleep apnoea symptoms & & & $<0.05$ \\
\hline No & $85(54.8)$ & $29(38.2)$ & \\
\hline Yes & $70(45.2)$ & $47(61.8)$ & \\
\hline $\begin{array}{l}\text { Duration of sleep prior to } \\
\text { work (hr) }\end{array}$ & & & $<0.05$ \\
\hline$<7$ & 99 (63.9) & $68(89.5)$ & \\
\hline$\geq 7$ & $56(36.1)$ & $8(10.5)$ & \\
\hline Rating of quality of sleep & & & $<0.05$ \\
\hline Very poor/poor & $27(17.4)$ & $53(69.7)$ & \\
\hline Average/good/very good & $128(82.6)$ & $23(30.3)$ & \\
\hline
\end{tabular}

*As 6 of the 231 taxi drivers declined height and weight measurements, body mass index was available for only 225 taxi drivers. Out of the 225 taxi drivers, 150 had an Epworth score < 10, and 75 had an Epworth score $\geq 10$. ITE: Institute of Technical Education; NS: nonsignificant
Table IV. Multiple logistic regression analysis of the association of risk factors with fatigue among taxi drivers, as graded using the Epworth Sleepiness Scale.

\begin{tabular}{|c|c|}
\hline Characteristic & Adjusted OR $(95 \% \mathrm{CI})$ * \\
\hline \multicolumn{2}{|l|}{ Age group (yr) } \\
\hline $31-50$ & 1 \\
\hline $51-70$ & $1.8(0.6-5.1)$ \\
\hline \multicolumn{2}{|l|}{ Body mass index ${ }^{*}\left(\mathrm{~kg} / \mathrm{m}^{2}\right)$} \\
\hline$<27.5$ & 1 \\
\hline$\geq 27.5$ & $2.4(0.9-6.6)$ \\
\hline \multicolumn{2}{|l|}{ No. of caffeinated drinks per day ${ }^{+}$} \\
\hline $0-2$ & 1 \\
\hline$\geq 3$ & $2.6(1.2-6.1)$ \\
\hline \multicolumn{2}{|l|}{ Shift status } \\
\hline Afternoon/overnight/24-hr shift & $2.1(0.5-8.3)$ \\
\hline Other shift timings & 1 \\
\hline \multicolumn{2}{|l|}{ No. of rest days per week } \\
\hline None & $1.2(0.5-3.0)$ \\
\hline$\geq 1$ & 1 \\
\hline \multicolumn{2}{|l|}{ Duration of driving per day $(\mathrm{hr})$} \\
\hline$\leq 10$ & 1 \\
\hline$>10$ & $1.3(1.0-1.7)$ \\
\hline \multicolumn{2}{|l|}{ Duration of each rest break (min) } \\
\hline$\leq 20$ & 1 \\
\hline$>20$ & $1.6(0.5-5.5)$ \\
\hline \multicolumn{2}{|l|}{ Additional part-time job ${ }^{+}$} \\
\hline No & 1 \\
\hline Yes & $7.5(1.7-32.9)$ \\
\hline \multicolumn{2}{|l|}{ Triad of sleep apnoea symptoms } \\
\hline No & 1 \\
\hline Yes & $1.1(0.5-2.7)$ \\
\hline \multicolumn{2}{|l|}{ Duration of sleep prior to work (hr) } \\
\hline$<7$ & $2.5(0.7-8.7)$ \\
\hline$\geq 7$ & 1 \\
\hline \multicolumn{2}{|l|}{ Rating of quality of sleep ${ }^{+}$} \\
\hline Very poor/poor & $8.2(3.0-22.5)$ \\
\hline Average/good/very good & 1 \\
\hline
\end{tabular}

*Adjusted for gender, educational level, ethnicity, age group, body mass index, number of caffeinated drinks daily, shift status, number of rest days weekly, duration of driving per day, duration of each rest break, additional part-time job, triad of sleep apnoea symptoms, duration of sleep prior to work and rating of

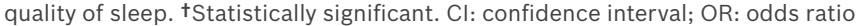

work, absence of rest days per week, $\leq 2$ rest breaks per shift, $\leq 20$ minutes per rest break, presence of a triad of sleep apnoea symptoms and $<7$ hours of sleep prior to work were not found to be associated with a statistically significant increase in fatigue and sleepiness, as shown in the adjusted odd ratios (Table IV).

\section{DISCUSSION}

In the present study, we found that the proportion of taxi drivers with self-reported medical conditions (i.e. hypertension, diabetes mellitus and high cholesterol) was much higher than that of the general Singapore population, as reported in the 2010 Singapore NHS. ${ }^{(16)}$ However, data from the 2010 NHS included Singapore residents aged between $18-69$ years, while $56.7 \%$ of the present study's population was aged between 51-70 years. As these medical conditions are age-related, the higher prevalence rates observed in the present study are expected. Another possible explanation for the observed higher rates of medical conditions 
is the observed unhealthy lifestyle habits of taxi drivers, i.e. the taxi drivers in the present study were found to smoke more, exercise less and have higher BMIs, as compared to the general population (based on NHS data) ${ }^{(16)}$ (Tables I \& II). As taxi drivers often spend long hours driving and have irregular schedules, they are more likely to have a physically sedentary lifestyle and limited food options (while on the road). This results in a higher risk of developing these lifestyle-related medical conditions as compared to the general population. The significantly high proportion of taxi drivers with medical conditions makes them a high-risk group for developing complications associated with these medical conditions. Also, it should be noted that the prevalence of medical conditions was likely to be under-reported in the present study, as the drivers might be undiagnosed, as was shown for the prevalence of hypertension, diabetes mellitus and high cholesterol among the general Singapore population in the 2010 NHS. ${ }^{(16)}$

To become a taxi driver in Singapore, drivers aged $\leq 50$ years have to undergo a medical check-up every two years, while drivers aged $>50$ years have to undergo an annual medical checkup. The medical check-up mainly consists of chest radiography, a visual acuity and colour perception test, medical history report and general medical examination by a doctor. While hypertension may be detected during the medical check-up, other chronic diseases such as diabetes mellitus and high cholesterol may not. The inability of the medical check-up to detect these diseases may pose a hidden risk to not only the taxi drivers, but also their passengers and other road users. This is because, if these comorbidities are not detected early, they may result in the occurrence of a stroke or an acute myocardial infarction during the course of work.

While poor ratings of quality of sleep were strongly associated with higher Epworth scores in the present study, the duration of sleep prior to work per day was not. Although the average sleep requirement is around $7-8$ hours per day for adults, most can tolerate a certain amount of partial sleep deprivation and still perform reasonably well. It appears that the limit of tolerance for fatigue is about 4-5 hours of sleep a day. ${ }^{(11)}$ Time of the sleep is also important in determining the magnitude and presence of fatigue. ${ }^{(11)}$

In the present study, the number of rest breaks per shift was not found to be negatively associated with fatigue. Although research has shown that rest breaks have an important restorative function, it also showed that the performance functions are unlikely to be restored to the initial levels and rest breaks are not known to restore performance levels after about 9 hours of work. ${ }^{(11)}$ Such findings have led to the restriction of driving hours to 9-10 hours per day by the International Labour Organization; this restriction is practised in both the United States and the United Kingdom. In the present study, driving more than 10 hours per day was noted to be a significant risk factor leading to fatigue, which is consistent with the results of studies conducted overseas. ${ }^{(11,12)}$

Although previous studies have shown that increasing age is a contributory cause of fatigue, ${ }^{(7)}$ this was not observed in the present study. A local study conducted about 30 years ago to examine the fatigue level among younger and older local taxi drivers did not show any correlation between age, and work capacity and accident liability. ${ }^{(18)}$

Driving the afternoon/overnight/24-hour shift was not shown to be a significant risk factor for sleepiness and fatigue among taxi drivers in the present study, although this shift status has been firmly established as a cause of fatigue among drivers in overseas studies. ${ }^{(11,12)}$ This difference may be due to the driver's personal adaptation to the shift status, environment and domestic conditions, after having worked for an extended period of time. ${ }^{(11)}$ Further studies may be required in order to conclusively determine the effects of shift status on fatigue among taxi drivers in Singapore.

The present study has several strengths. It is the first local study that specifically evaluated the risk factors of fatigued driving among taxi drivers, and thus, the results could be used as a baseline for future studies. Also, due to the cross-sectional nature of the study, we were able to simultaneously examine multiple risk factors of interest that were associated with fatigued driving. Furthermore, we were able to obtain the data required for analysis in a relatively short time and at a low cost.

However, despite the aforementioned strengths, the present study was not without limitations. One of the main limitations was participation bias. The response rate in the present study was $68.2 \%$, and those who declined to participate may have had a different demographic distribution from those who agreed to participate. We noted that some of the taxi drivers who declined to participate were resting on the sofas/chairs available at the company. In other words, the lack of participation from this group of drivers may have resulted in the underestimation of the prevalence of fatigue in the present study. Providing taxi drivers with incentives for participation such as complimentary food/drink coupons and/or blood/urine tests, securing greater assistance from the taxi company such as having notices of the study posted one month in advance, and translating the questionnaire into Malay and Tamil could have improved the participation rate. In addition, a larger sample size and a higher response rate across different taxi companies would have garnered better results and improved our understanding of the prevalence of fatigue and its associated risk factors.

Another possible limitation is recall bias. As the present study was a self-reporting survey, it is possible for the taxi drivers to underestimate their sleepiness and fatigue levels when scoring the ESS. This would have led to an underestimation of the prevalence of fatigue among the participants. Finally, the use of symptoms alone as an indicator of OSA may have resulted in a misclassification bias, as the use of a questionnaire survey to diagnose OSA has low sensitivity and specificity as compared to the use of polysomnography, which is currently the gold standard for diagnosis for OSA. ${ }^{(19)}$ Such a misclassification bias would have affected the estimation of associated adjusted odd ratios.

The present study found that a large proportion of taxi drivers had chronic medical conditions and unhealthy lifestyle habits such as smoking and physical inactivity. Effort should be made to promote a healthier lifestyle in this high-risk group, so as to curb the development of medical conditions and to prevent further 
complications from existing chronic medical conditions, if any. Targeted health promotion campaigns to raise awareness can be conducted in this high-risk group. Regular health screenings at subsidised rates can also be organised at taxi companies or medical clinics to allow early detection of any chronic diseases. Taxi drivers with one or more chronic diseases could be offered subsidised rates for treatment at medical clinics in order to encourage regular follow-up and better management of diseases and their associated complications.

Most local taxi drivers have to work long hours with few rest breaks during their work shift, as noted in the present study. It is our hope that our findings will help to raise awareness about the prevalence of fatigue among local taxi drivers and the risk factors leading to fatigue. Courses on how to recognise fatigue and its associated risk factors can be conducted during orientation programmes for new taxi drivers to increase awareness. Seminars on the dangers of fatigued driving can be also organised on a regular basis as a form of reminder for taxi drivers. Guidelines and legislation may also be passed as a form of enforcement. It may be necessary for government agencies, taxi companies and the National Taxi Association to work cooperatively to review existing policies and to incorporate appropriate guidelines on countermeasures to fatigued driving (e.g. the implementation of lessons on early recognition of fatigue for taxi drivers).

In conclusion, the demand for public transport in Singapore has been increasing due to expectations of a growing population. This has resulted in an increase in the number of taxis over the years, and the prevalence of fatigue among taxi drivers is certainly a concern for all, as fatigue affects the safety of not only the drivers, but also the passengers and other road users. With the appropriate measures put in place to address the health and working conditions of taxi drivers, we hope that a healthier and happier workforce of taxi drivers can be achieved. This will lead to a lower risk of road traffic accidents, which will in turn result in decreased economic loss, increased productivity and safer roads for all.

\section{ACKNOWLEDGEMENTS}

The present study was supported by the Practicum Fund, during the course of Master of Public Health at Saw Swee Hock School of Public Health, National University of Singapore. The authors would like to thank the taxi company and all the taxi drivers who participated in the study.

\section{REFERENCES}

1. Workplace safety and health guidelines - fatigue management. Ministry of Manpower and WSH Council, 2010. Available at https://www.wshc. sg/files/wshc/upload/cms/file/2014/Fatigue_Management.pdf. Accessed January 30, 2013.

2. Staal MA. Stress, Cognition, and Human Performance: A literature review and conceptual framework. National Aeronautics and Space Administration, August 2004. Available at http://human-factors.arc.nasa. gov/flightcognition/Publications/IH_054_Staal.pdf. Accessed January 30, 2013.

3. Connor J, Norton R, Ameratunga S, et al. Driver sleepiness and risk of serious injury to car occupants: population based case control study. BMJ 2002; 324:1125.

4. Drobnich D, A National Sleep Foundation's conference summary: the National Summit to Prevent Drowsy Driving and a new call to action. Ind Health 2005; 43:197-200.

5. McCartt AT, Ribner SA, Pack Al, Hammer MC. The scope and nature of the drowsy driving problem in New York State. Accid Anal Prev 1996; 28:511-17.

6. Beaulieu JK. The issues of fatigue and working time in the road transport sector. International Labour Office,Geneva, 2005. Available at http:// www.ilo.org/wcmsp5/groups/public/---ed_dialogue/---sector/documents/ publication/wcms_161410.pdf. Accessed January 31, 2013.

7. Di Milia L, Smolensky MH, Costa G, et al. Demographic factors, fatigue, and driving accidents: An examination of the published literature. Accid Anal Prev 2011; 43:516-32.

8. Wiegand DM, Hanowski RJ, McDonald SE. Commercial drivers' health: a naturalistic study of body mass index, fatigue, and involvement in safetycritical events. Traffic Inj Prev 2009; 10:573-9.

9. Smolensky MH, Di Milia L, Ohayon MM, Philip P. Sleep disorders, medical conditions, and road accident risk. Accid Anal Prev 2011; 43:533-48.

10. Winston AP, Hardwick E, Jaberi N. Neuropsychiatric effects of caffeine. Adv Psychiatr Treat 2005; 11:432-9.

11. Brown ID. Driver fatigue. Hum Factors 1994; 36:298-314.

12. C153 - Hours of work and rest periods (Road Transport) Convention, 1979 (No. 153). In: International Labour Organization [online]. Available at http://www.ilo.org/dyn/normlex/en/f?p=NORMLEXPUB:12100:0::NO: :P12100_ILO_CODE:C153. Accessed February 1, 2013.

13. Johns MW. A new method for measuring daytime sleepiness: the Epworth sleepiness scale. Sleep 1991; 14:540-5.

14. Chen NH, Johns MW, Li HY, et al. Validation of a Chinese version of the Epworth sleepiness scale. Qual Life Res 2002; 11:817-21.

15. Revision of Body Mass Index (BMI) Cut-Offs In Singapore. Health Promotion Board, Singapore, 16 March 2005. Available at http://www.hpb.gov.sg. Accessed February 10, 2013.

16. National Health Survey 2010, Singapore.Epidemiology \& Disease Control Division, Ministry of Health, Republic of Singapore, 2011. Available at https://www.moh.gov.sg/content/moh_web/home/Publications/ Reports/2011/national_health_survey2010.html. Accessed February 10, 2013.

17. Monthly Digest of Statistics Singapore, January 2013. Department of Statistics, Ministry of Trade \& Industry, Republic of Singapore, 2013. Available at http://www.singstat.gov.sg. Accessed February 10, 2013.

18. Koh D, Ong CN, Phoon WO. Effects of ageing on taxi driving. Ann Acad Med Singapore 1987; 16:106-9.

19. Management of Obstructive Sleep Apnoea/Hypopnoea Syndrome in Adults. Scottish Intercollegiate Guidelines Network, 2003. Available at http://www. sign.ac.uk/pdf/sign73.pdf. Accessed February 10, 2013 